\title{
Implementación de herramientas de control de calidad en MYPEs de confecciones y aplicación de mejora contínua PHRA
}

María PÉrez GaO Montoya ${ }^{1}$

RECIBIDO: 11/01/2017 ACEPTADO: 18/05/2017

\begin{abstract}
RESUMEN
Este articulo trata de una empresa dedicada, a la exportación prendas, que terceriza los procesos desde el tejido hasta el acabado. El tema se centra en el outsourcing de confecciones, qué es una MYPE y de su problema con respecto a la calidad.

La MYPE de confección no cuenta con un sistema de calidad definido, por lo que se plantea implementar herramientas de control de calidad y mejora continua para mejorar el nivel de calidad de los productos (prendas). Para la implementación de este trabajo se capacitó al personal en herramientas de control de calidad, mejora continua y método de autocontrol. Se realizaron mediciones antes y después de la implementación de herramientas. Se pudo conseguir una reducción en los niveles de fallados por confección de la MYPE.
\end{abstract}

Palabra clave: Herramientas de Calidad; Mejora Continua; MYPE

IMPLEMENTATION OF QUALITY CONTROL TOOLS IN CONFECTION MYPES AND CONTINUOUS IMPROVEMENT PDCA APPLICATION

\section{ABSTRACT}

This article is about a company dedicated to export cloths, which outsource the process from the tissue to the garment finishing. The topic is centered in confections outsourcing, what is a MyPE and their problem respect to quality.

Confections MyPEs do not count with a defined quality system, reason why the implementation of quality tools and continuous improvement to enhance their products (garment) quality standards is proposed. To implement this concept, the MyPE's staff was trained to use quality control tools, continuous improvement tools and the auto control method. Measurements pre and post the implementation of the mentioned tools were taken. A reduction of the confection error rate of the MyPE was achieved.

Keywords: Quality Tools; Continuous Improvement; MyPE

\section{INTRODUCCIÓN}

Dada la importancia de la calidad y teniendo en cuenta que la empresa exportadora de prendas terceriza (outsourcing en inglés) todos sus procesos, se plantea un procedimiento de calidad de uso sencillo para ayudar a las MYPES de confecciones a mejorar sus calidad y por ende sus procesos; teniendo en cuenta que estas carecen de personal técnico de control de calidad.

Según Brown \& Wilson (2005); outsourcing es el acto de obtener servicios de una fuente externa.

Según la "Ley MYPE”: MYPE es la unidad económica constituida por una persona natural o jurídica que considera los siguientes parámetros:

- Micro empresa abarca hasta 10 trabajadores y sus ingresos hasta 150 UIT.

- La Pequeña empresa abarca hasta 100 trabajadores y sus ingresos hasta 1700 UIT

El Ministerio de la Producción (2015), informa que la estructura empresarial formal del Perú es: Microempresa 95\%, Pequeña Empresa $4.3 \%$, Mediana empresa $0.2 \%$ y Gran Empresa $0.5 \%$.

Uno de los principales problemas que encuentran las empresas exportadoras de confecciones cuando contrata a las MYPES de confecciones, es el atraso en las entregas de la producción y el bajo nivel de calidad.

En todo proceso es muy importante contar con indicadores que le permita evaluar el desempeño, logro de metas y objetivos (Mondragón, 2002).

Como objetivo de la investigación se ha tomado el bajo nivel de calidad de las MYPES. Medir el nivel de calidad que genera sus procesos, es un punto de partida para implementar las mejoras. En las empresas de confecciones para la exportación, los clientes están evaluando contantemente los niveles de calidad de las empresas que contratan; y si estos exceden ciertos parámetros son penalizados y hasta se corre el riesgo de interrumpir el contrato de exportación.

1 Consultor Independiente.

Email : iperezgao@gmail.com 
En el presente trabajo se planteó implementar herramientas de Control de Calidad, Mejora Continua en las MYPES de confección, para mejorar el nivel de calidad en las órdenes de fabricación (disminuir el nivel de reprocesos).

\section{ANTECEDENTES}

Se tiene el trabajo de Mejora de un Proceso de Teñido en una Empresa Textil donde Briceño (2013) analiza los problemas de calidad de una empresa que terceriza todos sus procesos y concentra su estudio en los outsourcing de teñidos, proponiendo estándares y un sistema de control para estas empresas. Por otro lado el trabajo de Villaverde (2012) habla de la propuesta de implementación de los 14 principios de Deming en una empresa de envases, y la aplicación de Mejora continua. Ambos casos están orientadas a empresas de gran capacidad productiva. En el presente trabajo se plantó crear un sistema de control y mejora continua en una MYPE que no cuentan con un departamento de ingeniería, de proyectos ni de control de calidad. Se propone una aplicación sencilla de fácil manejo para el dueño del taller, el supervisor y la inspectora de calidad, herramienta que les permita medir su nivel de calidad y a partir de allí plantear mejoras para disminuir los reprocesos.

\section{MARCO TEORICO}

Ichikawa (1986), propone siente herramientas básicas e indispensables para el control de calidad, herramientas de fácil uso que no necesitan matemática avanzada, por lo que cualquier persona que se le instruya será capaz de utilizar. Estas herramientas son:

- Cuadro de Pareto

- Diagrama de causa y efecto

- Estratificación

- Hoja de verificación

- Histograma

- Diagrama de dispersión

- Gráficos de control

Deming (1989), propone elaborar una organización que guíe la mejora continua según el ciclo de Shewhart (también conocido como ciclo de Deming), que es un procedimiento muy valioso para quien quiere buscar una mejora, además asevera que en cualquier paso se puede necesitar metodología estadística para ir más rápido
Imai (2001) explica que la palabra Kaizen que es mejoramiento; en el lugar de trabajo tiene la connotación de mejoramiento continuo que involucra a todos y está orientada al proceso. Imai, indica que el "ciclo de Deming" o también llamado Ciclo PHRA (planificar, hacer, revisar, actuar), es una herramienta muy importante para la calidad y el mejoramiento continuo.

Donde:

Planificar: Significa hacer planes de los mejoramientos, usando herramientas estadísticas, tales como las siete herramientas: diagramas de Pareto, diagrama causa y efecto, histogramas, cartas de control, gráficas y lista de comprobación.

Hacer: es la aplicación del plan.

Revisar: verificar si se alcanzó la mejoría deseada.

Actuar: prevenir que se presente el problema, estandarizar.

De acuerdo a las investigaciones hechas por Suarez, et al. (2008) muestra los diferentes estudios sobre el kaizen bajo su versión japonesa y la versión de occidente más conocido como Mejora Continua, en donde se hace una aproximación a las prácticas japonesas indica que solo hay evidencia de la aplicación la mejora continua en grandes corporaciones de allí que existe una gran área de oportunidad de estudio en la pequeña y mediana empresa.

Rubinfeld (2011) plantea como transformar un grupo de trabajadores en un equipo de trabajo de alto rendimiento, plantea como formar y capacitar a los trabajadores en el logro de objetivos, como motivarlos, comprometerlos y formarlos para el autocontrol.

\section{METODOLOGÍA}

Se seleccionó una MYPE de Confecciones que tiene 18 trabajadores: un supervisor, un repartidor, 13 operarios de máquina, un mecánico a medio tiempo, 1 manual y 2 inspectoras. Se hizo una investigación aplicada, cuasi experimental en series de tiempo (Sánchez y Reyes, 2009) de un solo grupo. Siendo las duraciones del pre test y post test un mes cada uno. La población estuvo conformada por todas las órdenes de fabricación que se trabajaron en la MYPE que brinda servicios a la empresa exportadora. El estudio es probabilístico ya que se están tomando muestras al azar.

En el pre test se evaluaron 16 órdenes de fabricación por un total de 7985 prendas y en el post test, 17 órdenes de fabricación por un total de 8758 prendas. 


\subsection{Aplicación de la metodología}

Primero, se elaboró un formato para recabar la información, con la cual se generaron: los cuadros de control, se procedió a estratificar la información por grupos de máquinas, se hicieron los histogramas de las composturas generadas por cada máquina, diagrama de Pareto y diagramas de causa y efecto. Manejar la información de esta manera era más sencillo para las personas del taller de costura, que iba a trabajar con estas herramientas.

Se capacitó en herramientas básicas para el control de calidad (Cuadro de Pareto, Diagrama de Causa y Efecto, Histogramas y Gráfico de Control), tanto al supervisor, habilitador, inspectores, responsable del taller de confecciones, como a los auditores de la empresa exportadora.

Se capacitó en mejora continua, ciclo PHRA, al personal de la MYPE.

De la información procesada se obtuvo el indicador de total de fallados por costura, esto era el punto de partida, el objetivo era disminuir este porcentaje en forma gradual, aplicando herramientas de control de calidad y mejora continua (ver Cuadro 1 y Figura 1).

\section{Cuadro 1. Selección del problema a resolver}

TOTAL INPECCIONADO
\begin{tabular}{|l|c|c|c||c|} 
TOTAL FALLADO POR COSTURA & 2201 \\
PORCENTAJE & $\mathbf{2 7 . 6 \%}$ \\
\hline \hline & total & $\begin{array}{c}\% \\
\text { Absoluto }\end{array}$ & $\begin{array}{c}\text { Relativo } \\
\text { fallas }\end{array}$ & $\begin{array}{l}\% \text { Relativo } \\
\text { Acumulado }\end{array}$ \\
\hline Defectos & 1186 & $14.9 \%$ & $54 \%$ & $54 \%$ \\
\hline \hline Zurcido & 605 & $7.6 \%$ & $27 \%$ & $81 \%$ \\
\hline Compostura Costura Recta & 243 & $3.0 \%$ & $11 \%$ & $92 \%$ \\
\hline Compostura recubierto & 167 & $2.1 \%$ & $8 \%$ & $100 \%$ \\
\hline Compostura Remalle & 2201 & $27.6 \%$ & $100 \%$ & \\
\hline Total fallado & &
\end{tabular}

Fuente: Elaboración propia

Siendo los zurcidos composturas o reprocesos hechos a mano para solucionar recortes de puntadas y las composturas de costura recta, de recubierto o de remalle, son reprocesos hechos en sus respectivas máquinas para solucionar costuras mal ejecutadas.

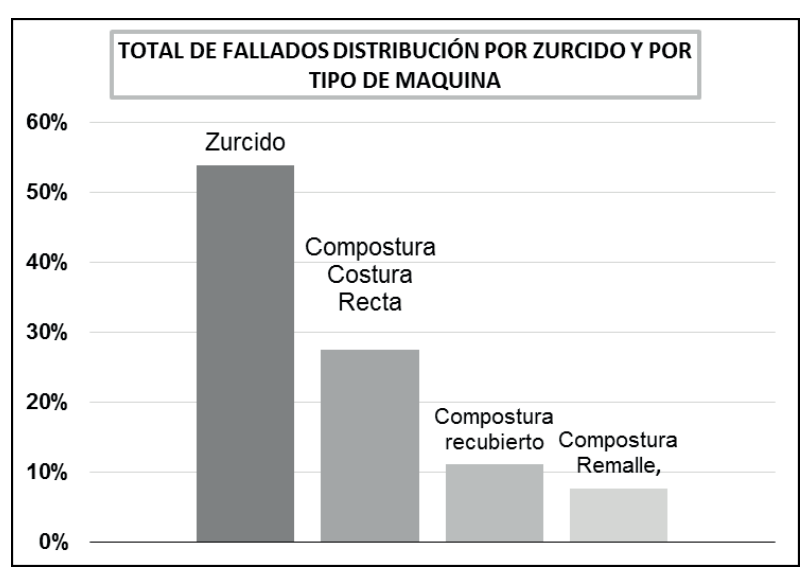

Figura 1. Distribución de fallas por tipo de máquina y zurcido Fuente: Elaboración propia

La información del Cuadro 1 y la Figura 1, sirve para visualizar los problemas encontrados (ambos tienen ordenados los defectos de mayor a menor) y priorizar los problemas por orden de importancia.

El siguiente paso fue reunir a los trabajadores para buscar las posibles causas de los problemas que se querían resolver (mediante lluvia de ideas). Del Cuadro 1 se puede apreciar que los 2 problemas principales que generaban el $81 \%$ de defectos (el zurcido y las composturas por costura recta).Por esta razón, éstos 2 problemas son los que deben priorizarse. En la Figura 2 se muestra el diagrama causa-efecto realizado en la presente investigación.

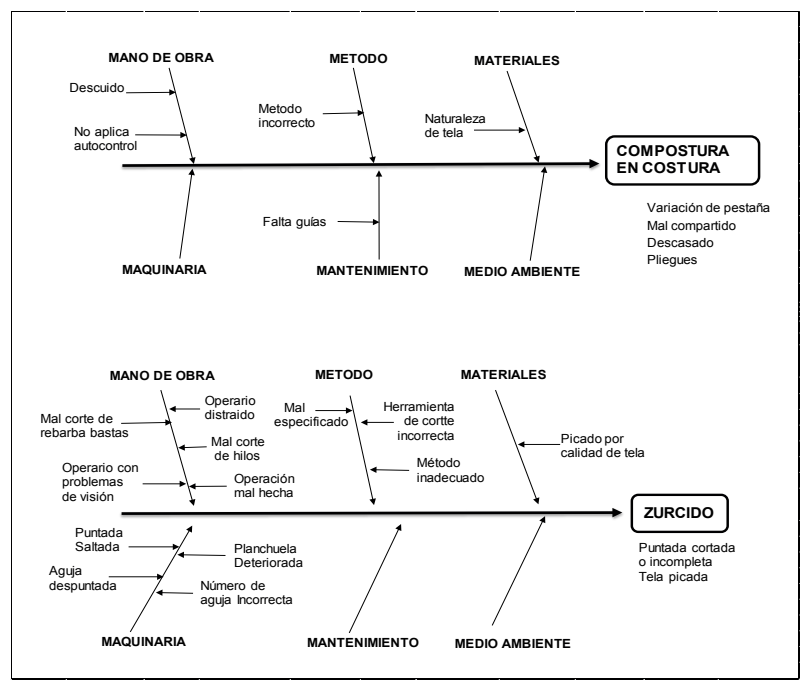

Figura 2. Análisis de la causa raíz del problema

Fuente: elaboración propia 
Una vez identificado el problema y sus posibles causas se propuso la siguiente meta: bajar el total de fallados de $27.6 \%$ a $23 \%$ (Figura 3 ) y se elaboró el plan de acción (Cuadro 2).

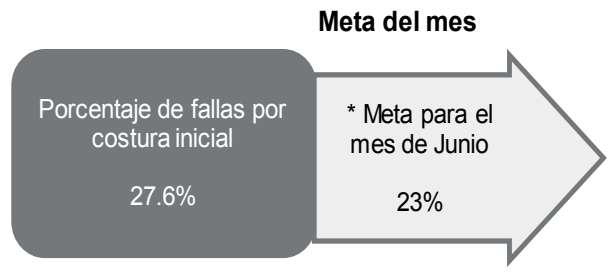

Figura 3. Meta del mes

Fuente: elaboración propia
Con la información analizada, se generó un plan de acción para implementar las soluciones. Esto se puede apreciar en el Cuadro 2, donde se tienen las actividades a implementar, los responsables y fechas de inicio y fin de actividades.

\section{RESULTADOS}

\subsection{Análisis Estadístico}

La escala de medición usada fue nominal, los estadísticos usados fueron frecuencia y conteo y el análisis inferencial fue a través de la prueba no paramétrica Chi cuadrado.

A continuación, se muestra la variable 1 donde se mide el nivel de reprocesos por costura en el taller en el pre test y post test.

Cuadro 2. Plan de actividades para bajar el porcentaje de fallas de costura

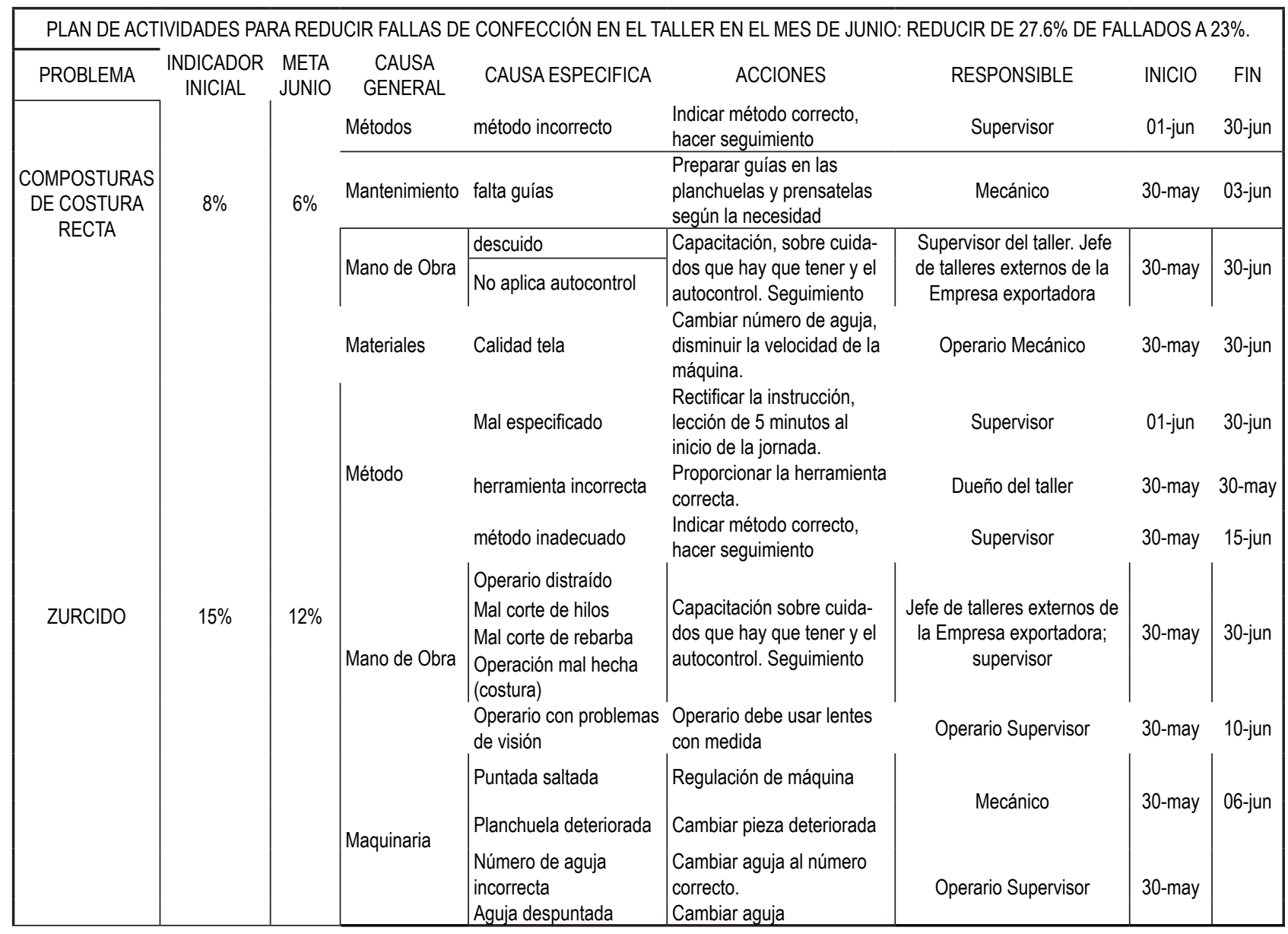

Fuente. Elaboración propia 
En el Cuadro 3, se muestra el cuadro de frecuencias del total revisado, el porcentaje de primeras y el nivel de reprocesos por costura en el pre y pos test.

Cuadro 3. Frecuencias pre y pos test variable 1

\begin{tabular}{|c|l|r|r|r|r|}
\cline { 3 - 6 } \multicolumn{2}{c|}{} & \multicolumn{2}{c|}{ Pre test } & \multicolumn{2}{c|}{ Pos Test } \\
\cline { 3 - 6 } \multicolumn{1}{c|}{} & Frecuencia & Porcentaje & Frecuencia & Porcentaje \\
\hline \multirow{3}{*}{ Valido } & $\begin{array}{l}\text { Primeras y } \\
\text { otros }\end{array}$ & 5784 & 72.4 & 6973 & 79.6 \\
\cline { 2 - 6 } & $\begin{array}{l}\text { Reprocesos } \\
\text { por costura }\end{array}$ & 2201 & 27.6 & 1785 & 20.4 \\
\cline { 2 - 6 } & Total & 7985 & 100.0 & 8758 & 100.0 \\
\hline
\end{tabular}

Fuente. Elaboración propia

En el Cuadro 3 se puede ver que el porcentaje de reprocesos del post test disminuyo de $27.6 \%$ a $20.4 \%$. Para probar estadísticamente esta afirmación se va a usar el contraste de hipótesis por chi-cuadrado para ver si hay o no una variación significativa en la disminución de nivel de reproceso.

A continuación se plantea las hipótesis estadísticas:

$\mathrm{H}_{0}$ : Sigue la distribución de $27.6 \%$ de reprocesos por costura y $72.4 \%$ primeras y otros.

$\mathrm{H}_{1}$ : No sigue la distribución de $27.6 \%$ de reprocesos por costura y $72.4 \%$ primeras y otros.

$\alpha: 0.05$

En el Cuadro 4 se puede ver que la frecuencia esperada es mayor a la observada, por lo que se podría decir que sí hubo una disminución de reprocesos en costura

Cuadro 4. Frecuencia Esperada varible 1 por chicuadrado

\begin{tabular}{|l|r|r|r|}
\hline \multicolumn{4}{|c|}{ Pos Test } \\
\hline & N observado & N esperada & Residuo \\
\hline $\begin{array}{l}\text { Primera y } \\
\text { otros }\end{array}$ & 6973 & 6340.8 & 632.2 \\
\hline $\begin{array}{l}\text { Reprocesos } \\
\text { de costura }\end{array}$ & 1785 & 2417.2 & -632.2 \\
\hline Total & 8758 & & \\
\hline
\end{tabular}

Fuente. Elaboración propia

Para validar esta conclusión revisemos la prueba chi cuadrado en el Cuadro 5.
Cuadro 5. Prueba chi-cuadrado para la hipótesis de la variable 1

\begin{tabular}{|l|r|}
\hline \multicolumn{2}{|c|}{ Estadísticos de prueba } \\
\hline & \multicolumn{1}{c|}{ Pos Test } \\
\hline Chi-cuadrado & $228,385^{\mathrm{a}}$ \\
\hline gl & 1 \\
\hline $\begin{array}{l}\text { Sig. } \\
\text { asintótica }\end{array}$ & .000 \\
\hline
\end{tabular}

Fuente. Elaboración propia

Como se puede ver en el Cuadro 5, "p" es menor que 0.001 ; consecuentemente "p" $<0.05$, por lo que se rechaza $\mathrm{H} 0$, y se acepta $\mathrm{H} 1$; se puede concluir que si hay una variación significativa en la disminución del nivel de reprocesos en las ordenes de fabricación confeccionadas en los talleres externos. La hipótesis H1 si se cumplió.

\section{CONCLUSIONES Y RECOMENDACIONES}

\subsection{Conclusiones}

- Se logró reducir el nivel de reprocesos de $27.6 \%$ a $20.4 \%$; como resultado del sistema propuesto.

- Beneficios para el taller de confección, al reducir el nivel de reprocesos, permitirá mejorar su productividad.

- Beneficios para la empresa exportadora, que disminuye sus gastos por arreglos, en órdenes aprobadas en el taller de confecciones.

- Beneficios para el taller de confecciones y la empresa exportadora al tener una producción más previsible, tanto por la medición de calidad como por el sistema de mejora continua que le permitirá ir disminuyendo el porcentaje de reprocesos.

\subsection{Recomendaciones}

- Las capacitaciones tienen que continuar, los primeros meses deben seguir con el apoyo del personal de la Empresa exportadora.

- Diariamente debe anotarse los resultados tantos de avance de producción como de calidad, en la pizarra para información de toda la línea.

- Mantener el sistema de información, para medir los avances y cumplimientos

- Trabajar con metas. 
- Hacer mantenimiento preventivo de las máquinas y equipo de producción.

- La empresa exportadora a través el encargado de talleres externos, deberá hacer seguimiento de los procedimientos implementados, mediante auditorias periódicas, y hacer reuniones periódicas con el encargado del taller y el supervisor.

- La Empresa exportadora, deberá aplicar los procedimientos aplicados en el taller de confecciones en estudio; en sus demás proveedores; de tal manera que se internalice los temas de calidad; y se cree el hábito de la mejora continua.

\section{BIBLIOGRAFIA}

[1] Briceño, I. (2013). Mejora de un Proceso Tercerizado: El Proceso de Teñido en una Empresa Textil. Sinergia E Innovación. Revisado 05, 9, 2015. Disponible en: http://revistas.upc.edu. pe/index.php/sinergia/article/view/155/123

[2] Brown, D., Wilson, S. \& Brown, P. (2005). The Black Book of Outsourcing. New Jersey, EEUU: WILEY.

[3] Cabrera, R. (2011). Lean Six Sigma TOC. Simplicado PYMES. Revisado el 01, 27,2015. Disponible en: https://books.google.com.pe/ books?id=psDDitEx_gC\&pg=PA63\&lpg=PA63\&d$q=e n t e n d e r+y+v e r+e l+m a p a+d e+l a+c a d e n a+d e+-$ valor\&source $=$ bl\&ots $=$ CZIOiiiz9j\&sig $=$ vZbL_ul3Td1FS004h8fjxdur1qc\&hl=es\&sa=X\&ei=xTCQVJe M8qwggTKvYDwBQ\&ved=0CC0Q6AEwAzgU\#$\mathrm{v}=$ onepage \&q=entender $\% 20 \mathrm{y} \% 20 \mathrm{ver} \% 20 \mathrm{el} \% 20$ mapa\%20de\%20la\%20cadena\%20de\%20valor\&$\mathrm{f}=$ false.

[4] Deming, E. (1989), Calidad, Productividad y competitividad, La Salida de la crisis. Madrid, España: Editorial de Santos S.A.

[5] Imai, M. (2001). Kaizen la clave de la Ventaja Competitiva Japonesa. (13 ed). México: Compañía Editorial Continental
[6] Ishikawa, K. (1986). ¿Qué es Control de Calidad Total? La modalidad Japonesa. Bogotá, Colombia: Editorial Norma.

[7] Ley MYPE D.S. No 007-2008-TR. Texto Único Ordenado de la Ley de Promoción de la Competitividad, Formación y Desarrollo de la Micro y Pequeña empresa y del Acceso al Empleo Decente. Diario el Peruano, Lima, Perú, 30 de setiembre de 2008

[8] Mondragón, A. (2009). Qué son los indicadores. Revisado 05, 17, 2015 Disponible en: http://www.orion2020.org/archivo/sistema_ mec/10_indicadores2.pdf

[9] Rubinfeid, H. (2011). El Juego del Trabajo. México: Editorial Pearson.

[10] Suarez, M. y Miguel, J. (2008). Encontrando al Kaizen: Un análisis de la Mejora Continua. Revisado 01, 22, 2015. Disponible en: https://buleria.unileon.es/xmlui/bitstream/handle/10612/1378/3117757\%5b1\%5d.pdf?sequence $\% 3 \mathrm{D} 1$

[11] Villarde, J. (2012). Propuesta de implementación de los 14 principios del Dr. Deming en una empresa de envases y envolturas plásticas (tesis maestría). Revisado 11, 26, 2014. Disponible en: http://tesis.pucp.edu.pe/repositorio/bitstream/handle/123456789/4478/VILLAVERDE_JESUS_PRINCIPIOS_DEMING. pdf?sequence $=1$.

[12] Ministerio de la Producción (2015). Anuario Estadístico Industrial, Mipyme y Comercio Interno 2015. Revisado: 10,02,2016 Disponible: http://www.produce.gob.pe/documentos/ estadisticas/anuarios/anuario-estadistico-mype-2015.pdf 